\title{
Nanoscopic surface inspection by analyzing the linear polarization degree of the scattered light
}

\author{
P. Albella,* J. M. Saiz, J. M. Sanz, F. González, and F. Moreno \\ Grupo de Óptica, Departamento de Física Aplicada, Facultad de Ciencias Universidad de Cantabria, \\ Avda de los Castros s/n, 39005 Santander, Spain \\ *Corresponding author: albellap@unican.es
}

Received January 7, 2009; revised May 13, 2009; accepted May 15, 2009; posted May 21, 2009 (Doc. ID 105799); published June 12, 2009

We present an optical method for the nanoscopic inspection of surfaces. The method is based on the spectral and polarization analysis of the light scattered by a probe nanoparticle close to the inspected surface. We explore the sensitivity to changes either in the probe-surface distance or in the refractive index of the surface. (C) 2009 Optical Society of America

OCIS codes: $290.5850,290.5855,240.6680,180.5810$.

Plasmonics is a recent research field with a great influence in nanotechnology [1,2]. During the past two decades, research in plasmonics involving metallic nanoparticles (NPs) has grown very fast, in part because of the need of different technological applications to inspect, monitor, and characterize materials, looking for particulate contaminants, roughness, and other defects in the subwavelength limit [3]. Around these applications, near-field microscopy techniques with nanoprobes have developed very fast. They enable to image phenomena, well beyond the diffraction limit (see [4] and references therein). The probe is key to the proper function of some near-field microscopes, in particular, those based on the spectroscopy of tips scanned along the sample [5,6]. Here is where these metallic NPs are of crucial importance owing to their size (resolution beyond the diffraction limit) [7] and the possibility of generating high intense fields on their surroundings (high sensitivity) [8]. Following the research line marked by the previous researchers, in a previous work [9] we presented a surface characterization method based on optical spectroscopy: a silver nanoparticle acting as a sensor was scanned over a surface. By studying the plasmonic shifts induced by the substrate, we were able to predict and characterize the existence of inhomogeneities on it. We propose in this Letter an optical inspection method based on the study of the linear polarization degree $P_{L}$ at normal incidence, defined as $(I s-I p) /(I s+I p)$ and measured at $90^{\circ}$, where $I s$ and $I p$ are the intensities with polarization perpendicular and parallel to the plane of incidence, respectively (see Fig. 1). In this scattering geometry small particles $(r=50 \mathrm{~nm})$ with $p$-polarized excitation do not radiate at $90^{\circ}$, and scattering, if any, is caused by the particle-surface interaction. Having this in mind, the system under study consists of a silver NP with cubic shape acting as a sensor and located close to a dielectric substrate. Both are illuminated with a plane wave linearly polarized in either the scattering plane or orthogonal to it, thus producing $I s$ and $I p$. This combined measurement can be very useful for profilometry and surface inspection, as it is sensitive to the NP-substrate distance and to the refractive index of the substrate.
The corresponding experimental arrangement is similar to that shown in Fig. 1(a) of [10], where the illumination of the probe can be made as in [4] in the far-field configuration. Figure 1 represents a model of a real experimental configuration.

The basic interaction mechanism arises from the fact that when a metallic NP is in the proximity of a substrate and both are under the action of an external electromagnetic field, the charge polarization induced on the particle causes another charge distribution on the substrate, which in turn also affects the NP. This charge distribution can be seen as the image charge distribution of the NP. Although for small NPs the dipolar approximation might be sufficient for describing the optical response of the isolated NP, the substrate-induced field acting on the whole volume of the NP, is no longer homogeneous in space, and multipolar modes of higher order might be excited in addition to the dipolar one [11]. The method we present here takes advantage of these multipolar modes that cause a variation in the linear polarization degree at $90^{\circ}$. All numerical calculations have been done using the discrete dipole approximation [12], which is a computational procedure that deals with the object by assuming that it is composed by a high enough number of elements, each one considered as a polarizable dipole.

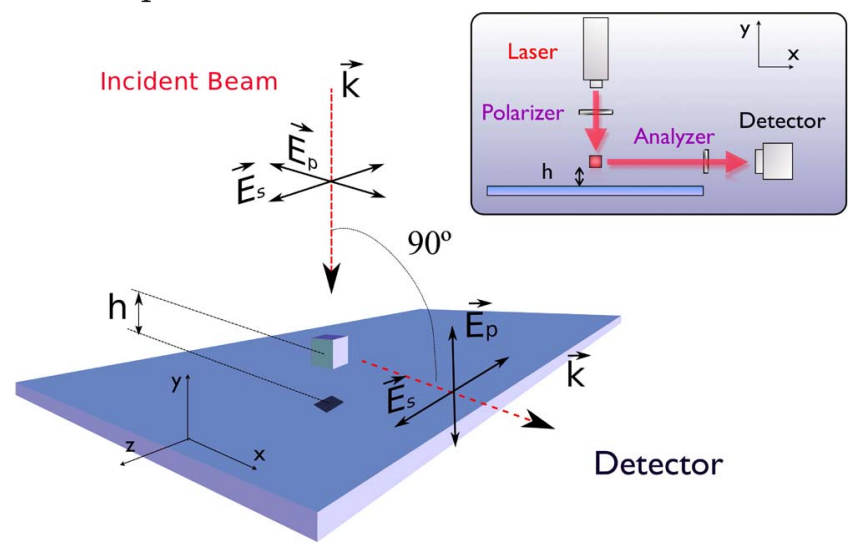

Fig. 1. (Color online) Scattering geometry. The inset shows a possible experimental arrangement, where the detector contains a long working distance microscope objective. 
For isolated light-scattering nanoparticles, we obtain in the small size limit the maximum value for the linear polarization degree at right-angle observation: $P_{L}=1$. This is due to the typical dipolar behavior of particles much smaller than the wavelength $(<50 \mathrm{~nm})$, producing no far-field scattering in the oscillation direction, but it does not hold for larger NPs, where other oscillation modes may be excited, as we will show later on. When a metallic particle approaches a substrate whose normal is in the same direction as the incident light beam, the system finds a natural limit in the isolated particle case when the $\mathrm{NP}$ is far enough from the substrate as to consider their interaction almost null. However, when the particle-substrate gap distance, $h$, is reduced, interactions, although being of the dipole-dipole kind, affect the scattering patterns, deforming the typical eight-shape of the near field of the isolated NP and producing some amount of $p$-polarized scattering [13]. As a result, the value of $P_{L}$ decreases from the unperturbed value of 1 . This effect is more notorious if the incident wavelength is able to excite some resonant oscillations or localized plasmons in the NP, being therefore very interesting to explore the spectral evolution of such parameter for a set of values of $h$. In Fig. 2 the spectral evolution of $P_{L}$ is shown for a silver cubic NP of size given by its side $L=40 \mathrm{~nm}$, above a dielectric substrate of refractive index $n=1.5$, and for distances for the particle-substrate gap ranging from $h=2 \mathrm{~nm}$ to $h=30 \mathrm{~nm}$. The limit case $(h \rightarrow \infty)$ is also included. Optical properties of silver have been taken from [14].

A small minimum of $P_{L}$ observed near $\lambda=400 \mathrm{~nm}$ for the isolated particle becomes a deep minimum over $\lambda=430 \mathrm{~nm}$ for $h=2 \mathrm{~nm}$. The value and position of the minimum are strongly dependent on the gap distance $h$, showing a redshift that increases with the interaction. This behavior is similar to that found for the scattered far field of resonant metallic NPs [11]. Also note that the plasmonic response of an NP depends strongly on its shape, making an interesting further study of how the NP shape affects the sensitivity and resolution of the proposed model. However,

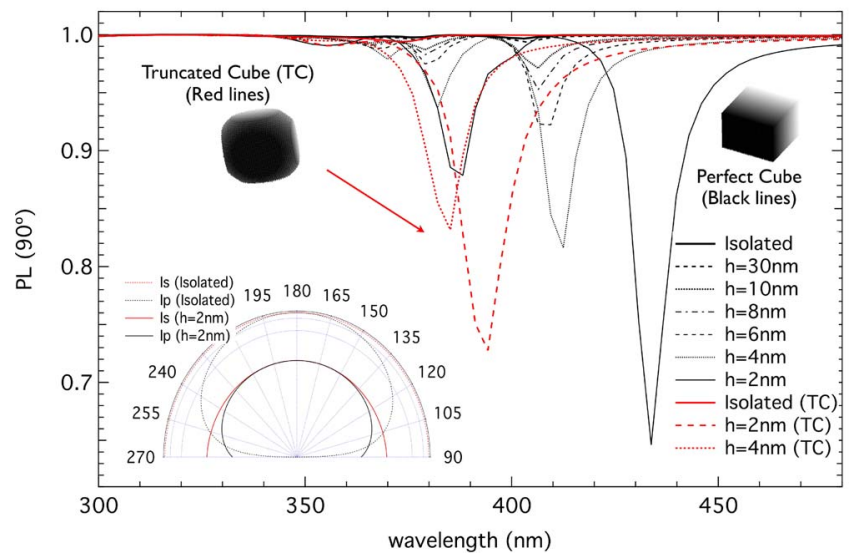

Fig. 2. (Color online) Spectrum of $P_{L}$ for a $40 \mathrm{~nm}$ NP located at different values of $h$. The inset shows the polar radiation diagram in log scale for an NP located at $h=8$ and $h=2 \mathrm{~nm}$, respectively. this study is out of the scope of this short communication. In Fig. 2 we have included, as an advance, the results obtained for a truncated cube (rounded vertex) located at $h=2 \mathrm{~nm}$ and $4 \mathrm{~nm}$.

Because the substrate is interacting with the particle and modifying the plasmonic resonant conditions, the spectral curves for $P_{L}$ necessarily have to depend on the dielectric constant of the substrate. In Fig. 3 some curves are shown for a cubic particle of $L=40 \mathrm{~nm}$, for a constant gap $h=4 \mathrm{~nm}$, and for a set of values of $n$, ranging from the limit case $n=1$ (isolated particle) to $n=2$. Again, enhancing the particle substrate interaction, this time by increasing the dielectric response of the medium, redshifts of the minimum of $P_{L}$ up to values over $\lambda=420 \mathrm{~nm}$ are observed. These dependences suggest the potential applications of $P_{L}$ measurement as a polarimetric tool for profilometry and surface inspection in conditions where the signal is weak (near grazing detection). In the case of profilometry application, the refractive index of the substrate needs to be known, whereas for the surface inspection application it is the tip-substrate distance, the parameter that has to be known. It is interesting to remark that very similar results were obtained when $P_{L}$ was calculated integrating over $10^{\circ}$ out of the $90^{\circ}$ direction, making possible to extend these results to scattering angles that separate a few degrees from grazing. This also gives a more realistic view of the possibilities of the method when taken to the experiment, where integration over small nonnull angular intervals is required.

In exploring the size dependence, our first group of results was connected with the checking of the mentioned dependences of $P_{L}$ for other values of $L=50 \mathrm{~nm}$. Similar trends were obtained for the series of $P_{L}$ curves, always with sharper minimum values for larger particles. This general behavior only confirmed the robust condition of a method based on the measurement of $P_{L}$ on a particle of small size.

The second group of calculations concerned NPs of size $L>50 \mathrm{~nm}$. Now, other modes of order higher than the dipolar appear, and even for the isolated particle, the $p$-polarized intensity is enhanced, and then $P_{L}$ tends to exhibit a very clear minimum. When the big NP approaches the substrate we find the results shown in Fig. 4. A silver cube sized $L=80 \mathrm{~nm}$ is

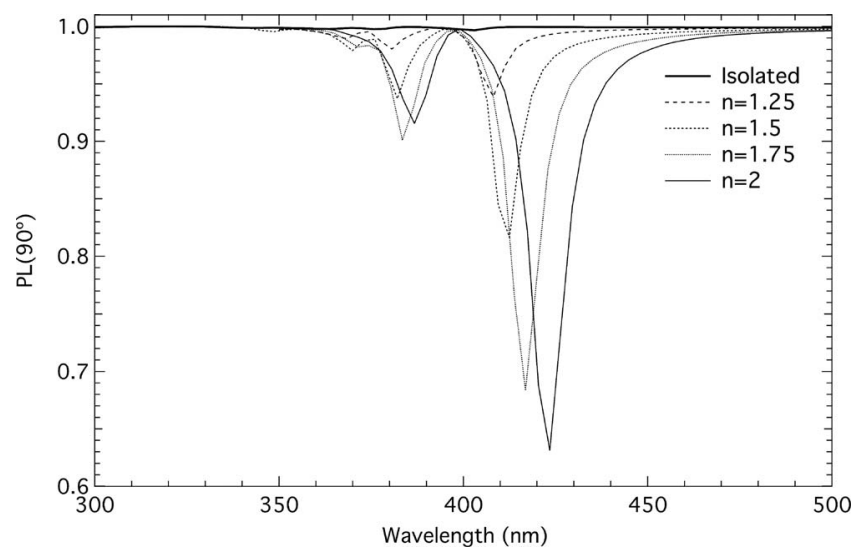

Fig. 3. Spectrum of $P_{L}$ for different substrate refractive indexes. 


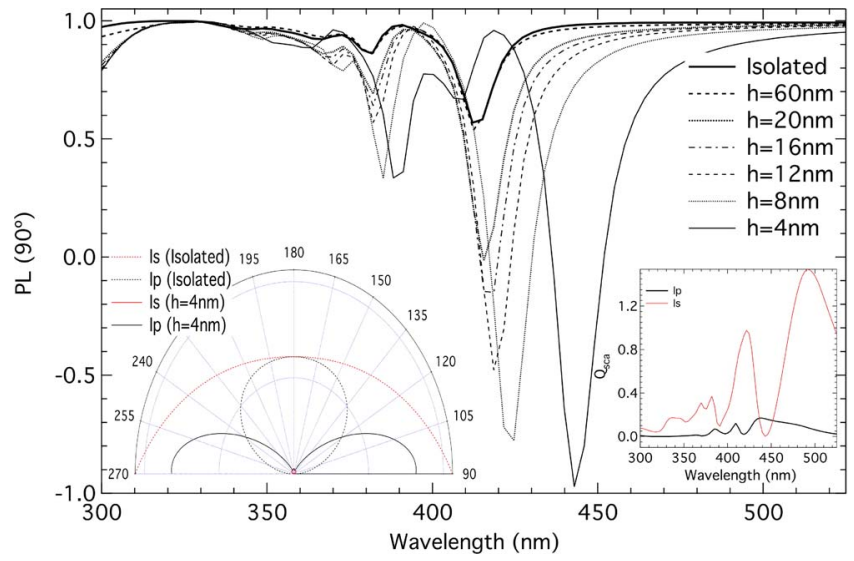

Fig. 4. (Color online) Spectrum of $P_{L}$ for a $80 \mathrm{~nm}$ nanocube located at different values of $h$. The inset shows the polar radiation diagram for an NP located at $h=8 \mathrm{~nm}$ and $h=4 \mathrm{~nm}$, respectively.

located above a dielectric surface $(n=1.5)$, with a gap ranging from $h=60 \mathrm{~nm}$ to $h=4 \mathrm{~nm}$. In this way $P_{L}$ is calculated for a set of curves that basically corresponds to scattering systems that are scaled by a factor of 2 with respect to the former set shown in Fig. 2. The redshift behavior of the minimum reaching values over $\lambda=440 \mathrm{~nm}$ for $h=4 \mathrm{~nm}$ and the increasing values of the minima are again found, but the most remarkable feature of these curves is the presence of intervals with $P_{L}<0$ obtained for $h<20 \mathrm{~nm}$.

This inversion in the sign of $P_{L}$ can be observed in the polar radiation pattern shown in the left inset of the figure. When the nanocube is close to the substrate, the eight-shape of the parallel component rotates $90^{\circ}$, therefore radiating in this direction. For completeness we show the parallel and perpendicular intensity spectrum in the right inset of Fig. 4, showing that this negative sign in $P_{L}$ is due to the enhancement of $I p$, which has higher values than $I s$ at certain wavelengths.

As a general conclusion, our proposal constitutes a technique complementary to that presented in $[5,6]$, where the measurement of spectral shifts of resonance peaks can give information about the surface. At this point, a comparison of both techniques (reso- lution, sensitivity, signal-to-noise ratio, etc.) would be very interesting in order to underline the main advantages of each one. However, this is out of the length for this Letter, and it can be a matter for more research on this topic.

This research has been supported by the Ministry of Science and Innovation of Spain under project FIS2007-60158 and U.S. Army International Technology Center-Atlantic through R\&D 1275-PH-01. The authors thankfully acknowledge the computer resources provided by the Red Española de Supercomputación node at Instituto de Fisica de Cantabria. P. Albella thanks the Ministry of Science and Innovation for his FPI grant.

\section{References}

1. E. Ozbay, Science 189, 311 (2006).

2. W. L. Barnes, A. Dereux, and T. W. Ebbesen, Nature 424, 824 (2003).

3. A. G. Davies and J. M. T. Thompson, Advances in Nanoengineering: Electronics, Materials and Assembly (Imperial College Press, 2007).

4. T. H. Taminiau, R. J. Moerland, F. B. Segerink, L. Kuipers, and N. F. van Hulst, Nano Lett. 7, 28 (2007).

5. T. Kalkbrenner, M. Ramstein, J. Mlynek, and V. Sandoghdar, J. Microsc. 202, 72 (2001).

6. T. Kalkbrenner, U. Håkanson, A. Schädle, S. Burger, C. Henkel, and V. Sandoghdar, Phys. Rev. Lett. 95, 200801 (2005).

7. H.-T. Chen, R. Kersting, and G. C. Cho, Appl. Phys. Lett. 83, 3009 (2003).

8. T.-J. Wang and W.-S. Lin, Appl. Phys. Lett. 89, 173903 (2006).

9. P. Albella, F. Moreno, J. M. Saiz, and F. González, Opt. Express 16, 12872 (2008).

10. K. G. Lee, H. W. Kihm, J. E. Kihm, W. J. Choi, H. Kim, C. Ropers, D. J. Park, Y. C. Yoon, S. B. Choi, D. H. Woo, J. Kim, B. Lee, Q. H. Park, C. Lienau, and S. Kim, Nat. Photonics 1, 53 (2007).

11. F. Moreno, F. González, and J. M. Saiz, Opt. Lett. 31, 1902 (2006)

12. B. T. Draine and P. J. Flatau, User Guide for the Discrete Dipole Approximation Code DDSCAT 6.1 (2004), http://arxiv.org/abs/astro-ph/0409262v2.

13. F. Moreno, B. García-Cámara, J. M. Saiz, and F. González, Opt. Express 16, 12487 (2006).

14. P. Johnson and R. Christy, Phys. Rev. B 6, 4370 (1972). 